\title{
New Feature-level Algorithm for a Face-fingerprint Integral Multi-biometrics Identification System
}

\author{
Bayan Omar Mohammed ${ }^{1}$, Hamsa D. Majeed², Siti Zaiton Mohd Hashim³, \\ Muzhir Shaban Al-Ani ${ }^{4}$ \\ ${ }_{1,2,4}$ Department of Information Technology, College of Science and Technology, University of Human Development, \\ Kurdistan Region, Iraq, ${ }^{3}$ Department of Data Science, Universiti Malaysia Kelantan (UMK), Taman Bendahara, 16100 \\ Pengkalan Chepa, Kelantan
}

\section{A B S T R A C T}

This article delves into the power of multi-biometric fusion for individual identification. a new feature-level algorithm is proposed that is the Dis-Eigen algorithm. Here, a feature-fusion framework is proposed for attaining better accuracy when identifying individuals for multiple biometrics. The framework, therefore, underpins the new multi-biometric system as it guides multi-biometric fusion applications at the feature phase for identifying individuals. In this regard, the Facefingerprints of 20 individuals represented by 160 images were used in this framework. Experimental resultants of the proposed approach show $93.70 \%$ identification rate with feature-level fusion multi-biometric individual identification.

Index Terms: Multi-model Biometric, Dis-Eigen algorithm, Identification, Aspect United Moment Invariant

\section{INTRODUCTION}

Technologies of biometric are utilized as a source in multiple applications that outline the interests of identification and authorization, such attentiveness demands a high level of privacy and security. Compared with traditional security, biometrics is considered an important type of security since it provides unique features through identifying biometrics characteristics [1].

A large number of security-based researchers used biometrics systems to improve the performance of their approaches. The biometric specifications are classified into two main classes: Physiological and behavioral, body parts included under fixed human characteristics and classified to be physiological

\begin{tabular}{|l|l|}
\hline Access this article online \\
\hline DOI:10.21928/undjst.v6n1y2022.pp12-20 & $\begin{array}{l}\text { E-ISSN: 2521-4217 } \\
\text { P-ISSN: 2521-4209 }\end{array}$ \\
\hline
\end{tabular}

Copyright (c) 2022 Mohammes et al. This is an open access article distributed under the Creative Commons Attribution Non-Commercial No Derivatives License 4.0 (CC BY-NC-ND 4.0) characteristics for instance iris, fingerprint, face, DNA, and retina, also classified as passive biometrics, while gait, voice, and handwritten signature classified as active biometrics as it represented by skills or functions performed by an individual and that make them belong to behavioral characteristics. Either way, those characteristics led to high authentication and verification for security [2].

Biometric security can be achieved through two kinds of categories: uni-model and multi-model [3]. Online list of biometric characteristics (active or passive) is used as a feature in the uni-model, this model has a low-security level against the multi-model which uses two or more of those characteristics that achieve a higher security level.

The main biometric characteristics for personal verification are obtained from face-fingerprint characteristics, the main aim of this paper is to improve human identification through the multi-model biometric process through merging between two biometric features, face-fingerprint, via these features, the system can compare, detect and identify the candidate within the constructed dataset. Both of these characteristics require

Corresponding author's e-mail: Bayan.omar@uhd.edu.iq, hamsa.al-rubaie@uhd.edu.iq , sitizaiton@umk.edu.my ,muzhir.al-ani@uhd.edu.iq 
the existence of reference biometric data samples taken from different volunteers of different ages to be compared against the respective biometrical data of every person enrolled in a database or against a single reference template of particular enrolled individuals for identity confirmation of that person respectively [4].

The identification system accuracy is determined by its success comparison relying on the uniqueness of people's biometric characteristics, i.e, two persons can never have the same features [1]-[4].

\section{RELATED WORK}

According to the importance of using biometric systems in different applications and implementations, this field attracted countless researchers to propose their approaches using different biometrics characteristics. This section presents the most recent published approaches in this field.

In fingerprint recognition orientation, several techniques are recommended for accuracy enhancement of the recognition, those techniques were different upon various criteria, certain of these proposals areas of interest was in the preprocessing stage of fingerprint images [5], implemented an approach for the features extraction of both right and left-hand thumbs using many levels of twodimensional discrete wavelet transform (2D-DWT), while another approach [6] used Discrete Cosine Transform (DCT) technique for extraction features [7], [8] presented techniques for fingerprint enhancement through localizing and recognizing the minutiae for minutiae extraction relying on the optimal thinning operation that took place in the preprocessing stage of fingerprint image. Prasad et al. [9] proposed a system consisting of many stages starting from data gathering which includes fingerprint images belonging to many different people then pre-processing those depending on their characteristics, finally, the algorithm is used for the purpose of recognition of fingerprint. Another area of interest was in proposing and developing algorithms for extracted feature stage from the process of recognition of that fingerprint, [10] proposed multi-biometric fusion for identical twins at the feature-level with Dis-Mean algorithm, Aspect United Moment Invariant (AUMI) used to define the individual biometric fingerprint characteristic. The extracted features regard the twin handwriting fingerprint for both word and shape. Furthermore, [11] proposed a fusion algorithm using the Mean-Discrete feature for identical twins fingerprint detection, The main method of the presented algorithm requires the person's class labeling and multimodel biometric features to uni-modal biometric features conversion. As in multi-modal biometrics, the individuality represented by Mohammed and Hashim [12] using (AUMI) for global feature extractions to serve as a means to identical twins fingerprint detection, the procedure of Individuality representation measures the AUMI capacity for the individuality of the main of twin handwriting-fingerprint. A modified algorithm proposed by Mohammed [13] for Individuality Representation by employing the Mean-Discrete Algorithm, the vector of feature carries the features which generalized the global features owned by individuals. The developed model generalized the features in an earlier stage of classification.

In face recognition, many approaches have been proposed over the years that compound pattern recognition with computer vision and image processing, those algorithms are implemented in many forms to accomplish high-efficiency recognition. Al-Shayea et al. [14] present algorithm to specify the recognition rate of the PCA algorithm before and after applying DWT. The outcome was that applying DWT increases the recognition rate with minimization in the feature matrix size. Paper [15] proposed an approach based on wavelet-curvelet for facial features extraction, the used technique aims to reduce mathematical computational image analysis by reducing the dimensionality. The Nearest Mean Classifier (NMC) is employed for recognition at a high rate. An efficient approach is proposed [16] for face recognition concentrated on the efficiency of the image to calculate the required features from the image that contained the face. The outcome result gives a fine performance of the face recognized from that system. Qeethara Al-Shayea [17] proposed a system for information measurement between the main features of different face parts like angles and distances. An algorithm has been constructed with all the digital data compared with massive face images databases. Histogram equalization technique is used for extraction feature for recognition. Research [18] has a main objective of designing an efficient face recognition approach by generating a matrix with a significant rank by applying the technique of singular value decomposition. A relative study is made by Prasad et al. [19] scientific survey generated to study various methods and techniques with all the sufferings and benefits of these approaches. Al Ani and Al Waisy [20] presented an approach using a kernel machine for face detection from different views, the proposed algorithm shows powerful ability to effectively multi-view face detection. Another proposal presented by Nejrs and Al-Ani [21] for face classification through a structured approach that is implemented using 
dissimilar levels in Two-Dimensional Discrete Fourier Transform (2D-DWT). While [22] proposed a technique to track faces and detect happiness parameters. The system is performed using a Raspberry Pi device, high-resolution camera, and high-definition screen. The study is compatible to apply in real-time. The main goal of this proposal is for studying the happiness level that the students have in the class and raise that level during the lecture.

Other researchers shared our interest in integrating facefingerprint recognition in one system. Sriram J and Jacob J [23] proposed a voting system EVM where all the data of voters are digitally recorded. Through the proposed project, the faces of the voters are recognized first by a camera then the fingerprint is used for giving authentication through the data that is already stored in the database. Another multimodal system was proposed [24] for identification using face-fingerprint features. The calculations are taking place in the work first for each feature individually rather than when they are combined together. Divyakant and Meva [25] proposed a biometric system for performance assessment of face-fingerprint biometric traits collected from 30 humans. The recognition stage performance is measured through(False Acceptance Rate) FAR and (False Rejection Rate) FRR calculations. A comb filter approach is proposed systems [26] regarding face-fingerprint recognition through an encryption process to present cancelable patterns then make a comparison with any other encrypted biometric that have been randomly attacked. In article [27], firstly in the part of novel step, a system of smart cards accessing is presented by face authentication first then by fingerprint concurrently. The verification should be for both mutual authentications, after that the transaction is approvable [12].

\section{THE MULTI-BIOMETRIC IDENTIFICATION SYSTEM APPROACH PROPOSAL}

With regards to getting a high accuracy identification, the classifier input is rich with the major features extraction of individuals. In this work, the features are extracted for individual face-fingerprint. After extraction, the classification takes place so as to improve biometric identification. In this module, the feature sets of the defined processed biometrics data are extracted. Feature extraction is crucial for resolving raw data into simple, clear, and comprehensible data that would be able for matching learning. This phase is crucial in almost all systems of pattern recognition. The extraction of global features for both face-fingerprints including the extraction of macro features and method of minutiae-based extraction.
The Aspect United Moment Invariant (AUMI) is extracted to attain the individual features from the images of individual face-fingerprint. The extracted features are all kept in the Storage of Invariant Feature Vector.

The proposed system for multimodal biometrics identification that employs two biometrics modalities is presented in Fig. 1. The adopted phase following feature extraction comprises the suggested fusion feature level.

At the level of feature fusion, signals that come from different biometrics channels are processed first. Meanwhile, feature vectors are separately extracted then go through a specific field algorithm of fusion known as the Dis-Eigen algorithm. These feature vectors are composite to generate a combined feature vector prior to being employed for similarity measurement and classification process.

\section{EXTRACTION OF THE FEATURE}

The feature sets are extracted from the defined processed biometrics data in this work using Aspect United Moment Invariant (AUMI) [28]. It is powerful in capturing the biometrics' individual global characteristics of Physiognomy and style of the fingerprint and for global features continuously and separately into an individual representation. The scorce of Aspect United Moment Invariant Structure is below and represented in Fig. 2.

Entirely There are eight AUMI features reported below:

$$
\begin{gathered}
\theta_{1}=\frac{\sqrt{\varnothing_{2}}}{\varnothing_{1}} \\
\theta_{2}=\frac{\varnothing_{6}}{\varnothing_{1} \varnothing_{4}} \\
\theta_{3}=\frac{\sqrt{\varnothing_{5}}}{\varnothing_{4}} \\
\theta_{4}=\frac{\varnothing_{3}}{\varnothing_{2} \varnothing_{4}} \\
\theta_{5}=\frac{\varnothing_{1} \varnothing_{6}}{\varnothing_{1} \varnothing_{3}} \\
\theta_{6}=\frac{\left(\varnothing_{1}+\sqrt{\varnothing_{2}}\right) \varnothing_{2}}{\varnothing_{6}}
\end{gathered}
$$




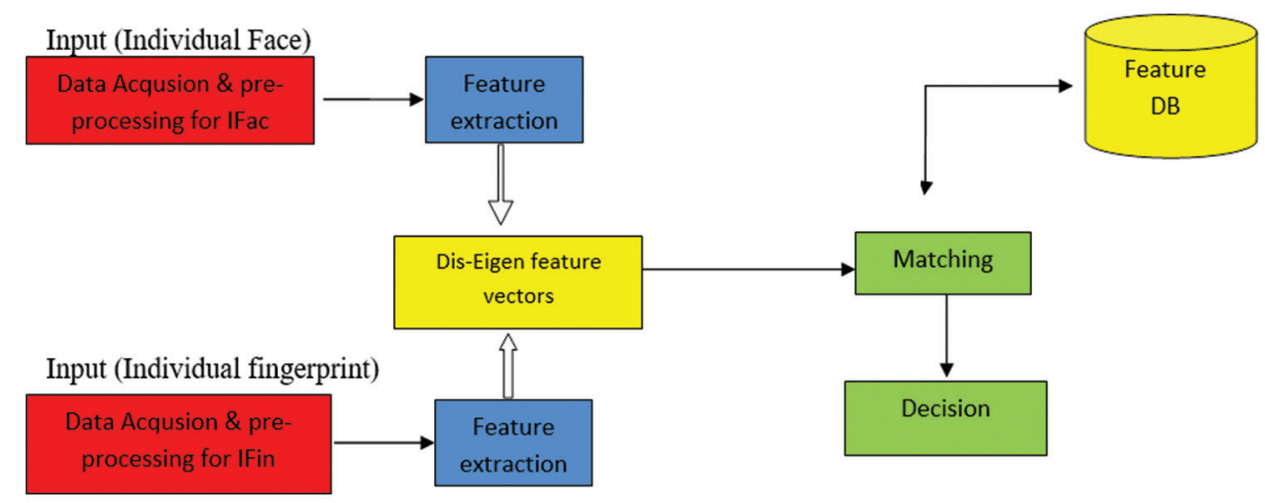

Fig. 1. Proposed multi-biometric system.

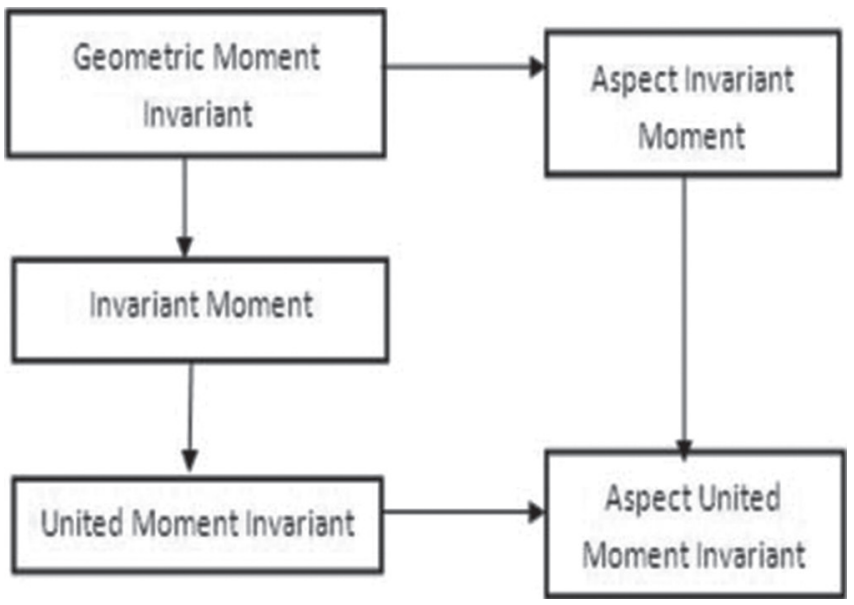

Fig. 2. Aspect united moment invariant structure.

$$
\begin{gathered}
\theta_{7}=\frac{\varnothing_{1} \varnothing_{5}}{\varnothing_{3} \varnothing_{6}} \\
\theta_{8}=\frac{\varnothing_{3}+\varnothing_{4}}{\sqrt{\varnothing_{3}}}
\end{gathered}
$$

Where $\varphi$ i represents Hues moment invariants. Due to the large values of $\varphi i$, the natural logarithm is applied thus giving us: for $\mathrm{i}=1$ to 7 ; $\theta \mathrm{i} \leftarrow \log 10 \varphi \mathrm{i}$.

A set of global features are generated from the extracted features. For the purpose of improving identification performance, these features are gathered individually. Since the extracted features are in the multirepresentations zone, then it has been used in combined form. Such combined features are termed as Dis-Eigen feature vectors in the Uni-representation zone, which is employed after the process of feature extraction [12], [28], [29].

\section{THE PROPOSED DISCRETIZED-EIGEN (DIS-EIGEN) ALGORITHM IN FEATURE LEVEL FUSION}

This work attempts to design a more effective multimodal biometrics identification system introduces by introducing the new proposed Dis-Eigen feature-based fusion as Fig. 3 illustrates with the capacity in generating distinctive features of numerous modalities of individuals, where fac and fin represent the face and fingerprint features consecutively. First, an improved AUMI is used as global in the extraction of features obtained from the individual face-fingerprint shape and style. Then, the features-based fusion is examined in terms of its generalization. Further, to achieve better classification accuracy, the Dis-Eigen feature-based fusion algorithm was used.

At the start of the process, Dis-Eigen feature-based fusion processes the raw biometrics input images using the suitable technique of processing. This results in images of standard size and quality. Then, the feature extraction algorithm is used to extract these images to generate discriminatory information which can provide a distinction between the identities. It is not easy to come up with a method that could comprehensively capture the discriminatory information from both raw input images among numerous identities or modalities and resolve problems in biometrics analysis. This study proposes the use of an enhanced combined

Feature vector fusion and uni-representation are known as the Dis-Eigen feature for multimodal biometrics identification. Also, Dis-Eigen replaces the feature fusion as a feature transformation agent to provide better feature representation from numerous modalities.

In the Dis-Eigen process, multi representation features from multi- biometrics are converted to uni- and systematic 
Mohammed et al.: Dis-Eigen algorithm in Feature-level Fusion

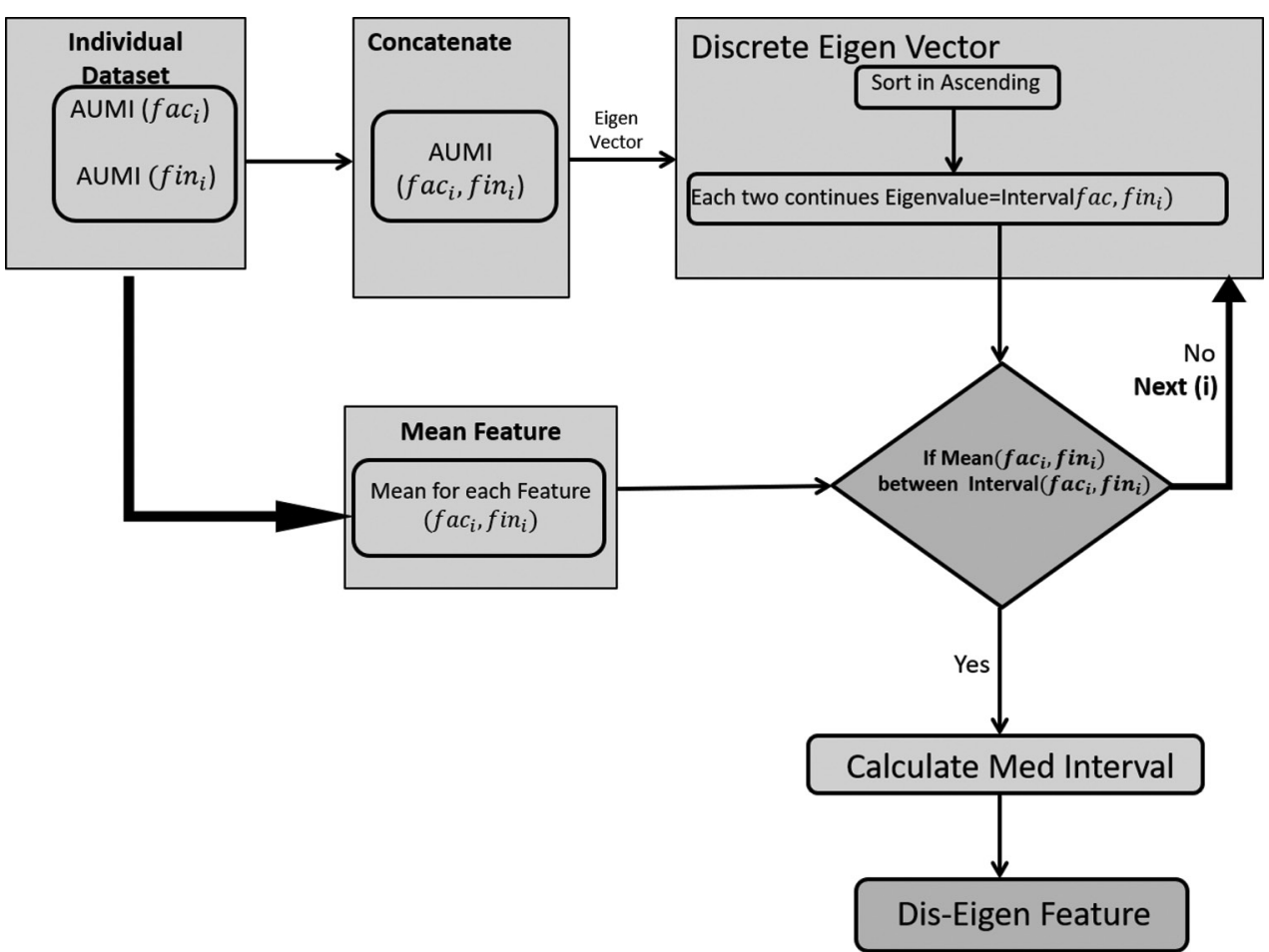

Fig. 3. The proposed dis-eigen feature-based fusion.

representation features for reduction of the complexity (dimension) of the feature vector. After concatenating an individual face-fingerprint the sorted Eigenvector in ascending order for a person in the aim of determining discrete intervals in the Dis-Eigen line. The dis-Eigen line is a line of an invariant feature vector that starts from the minimum Eigenvalue and ends with the maximum Eigenvalue from the Eigen feature vector for that person. The interval number is fit equally to the capacity of Eigenvector -1 .

The eight features columns of AUMI have been applied in this study. The eigenvalue is the divider of intervals in the Dis-Eigen line. Each mean Face-Fingerprint feature (FF) vector that falls within the same interval will have the same Med Interval value. Med Interval (MI) for each interval is the average of an interval that is calculated using the formula as in (9):

$$
M I=\frac{e v_{i}-e v_{i+1}}{2}
$$

Med Interval value for interval one to seven represents the invariant feature

vector that falls within if $F F \geq e v_{i}$ and if $F F \leq e v_{i+1}$. On the other hand, compute the mean features that come from the individual face ( $\mathrm{fac}_{\mathrm{ij}}$ ) and individual fingerprint ( $\mathrm{fin}_{\mathrm{ij}}$ ) invariant feature vector for a person in a twin. The computation and reduction for each face-fingerprint feature are expressed as below:

$$
F F_{i j}=\left(f a c_{i j}+f i n_{i j}\right) / 2
$$

Where:

$\mathrm{fac}_{i j}$ : face features for an individual.

fin $_{i j}$ fingerprint features for an individual.

Eight features are created in this study and this is the column number for the features number of the applied AUMI for multi-biometrics. These features are called the Dis-Eigen feature-based fusion vector.

Tables 1-3 below exemplify the transformation of individual multimodal biometrics feature vector into Dis-Eigen featurebased fusion vector:

Tables 1 comprise eight columns representing the eight columns of invariant feature vectors within the AUMI then concatenated. These data are further applied in the Dis-Eigen process.

While Table 2 represents the Dis-Eigen features vector composed of the generalized features of individual features for an individual. Meantime Table 3 presents Dis-Eigen 
Mohammed et al.: Dis-Eigen algorithm in Feature-level Fusion

TABLE 1: Real Data for face and fingerprint for individual number 1

\begin{tabular}{|c|c|c|c|c|c|c|c|c|}
\hline Image & F1 & F2 & F3 & F4 & F5 & F6 & F7 & F8 \\
\hline & 1.0075 & 2.6937 & 1.7301 & 0.3341 & 2.9618 & 3.3513 & 1.2401 & 5.7571 \\
\hline & 1.0075 & 5.2967 & 1.7303 & 0.334 & 5.8222 & 1.7048 & 630.6227 & 5.758 \\
\hline & 1.0054 & 5.7202 & 1.7308 & 0.3338 & 6.3065 & 1.5772 & 583.5766 & 5.7626 \\
\hline & 1.0178 & 0.1602 & 1.7237 & 0.3363 & 0.0175 & 56.1404 & 2.0994 & 5.7059 \\
\hline & 1.0142 & 0.1418 & 1.7242 & 0.3361 & 0.0156 & 63.2681 & 2.3703 & 5.71 \\
\hline & 1.0305 & 0.0901 & 1.7238 & 0.3363 & 0.0096 & 101.1057 & 3.7337 & 5.7062 \\
\hline & 1.0178 & 0.1021 & 1.7239 & 0.3362 & 0.011 & 88.8608 & 3.2916 & 5.7073 \\
\hline
\end{tabular}

TABLE 2: Dis-Eigen face and fingerprint for individual number1

\begin{tabular}{lcccccc}
\hline F1 & F2 & F3 & F4 & F5 & F6 & F7 \\
\hline 1.2312 & 1.2312 & 1.2312 & 1.2312 & 1.2312 & 57.6571 & 1.2312 \\
1.2312 & 1.2312 & 1.2312 & 1.2312 & 1.2312 & 57.6571 & 0 \\
1.2312 & 1.2312 & 1.2312 & 1.2312 & 1.2312 & 57.6571 & 1.2312 \\
1.2312 & 1.2312 & 1.2312 & 1.2312 & 1.2312 & 57.6571 & 1.2312 \\
\hline
\end{tabular}

TABLE 3: Example of dis-eigen feature for individuals

\begin{tabular}{|c|c|c|c|c|c|c|c|c|}
\hline F1 & F2 & F3 & F4 & F5 & F6 & F7 & F8 & Individual \\
\hline 3.4057 & 3.4057 & 3.4057 & 0.437 & 3.4057 & 47.802 & 0 & 3.4057 & P10 \\
\hline 3.4057 & 3.4057 & 3.4057 & 0.437 & 3.4057 & 47.802 & 0 & 3.4057 & P10 \\
\hline 3.4057 & 3.4057 & 3.4057 & 0.437 & 3.4057 & 47.802 & 3.4057 & 3.4057 & P10 \\
\hline 0.5237 & 4.5258 & 4.5258 & 0.5237 & 4.5258 & 54.7769 & 0 & 4.5258 & $\mathrm{P} 11$ \\
\hline 0.5237 & 4.5258 & 4.5258 & 0.5237 & 4.5258 & 54.7769 & 0 & 4.5258 & $\mathrm{P} 11$ \\
\hline 0.5237 & 4.5258 & 4.5258 & 0.5237 & 4.5258 & 54.7769 & 4.5258 & 4.5258 & P11 \\
\hline 1.2422 & 5.3632 & 1.2422 & 1.2422 & 5.3632 & 58.042 & 0 & 5.3632 & P12 \\
\hline 1.2422 & 5.3632 & 1.2422 & 1.2422 & 5.3632 & 58.042 & 58.042 & 5.3632 & P12 \\
\hline 1.2422 & 5.3632 & 1.2422 & 1.2422 & 5.3632 & 58.042 & 0 & 5.3632 & P12 \\
\hline 1.8191 & 6.8858 & 1.8191 & 1.8191 & 6.8858 & 64.3154 & 0 & 6.8858 & P13 \\
\hline 1.8191 & 6.8858 & 1.8191 & 1.8191 & 1.8191 & 64.3154 & 0 & 6.8858 & P13 \\
\hline 1.8191 & 1.8191 & 1.8191 & 1.8191 & 1.8191 & 64.3154 & 0 & 6.8858 & P13 \\
\hline 1.7582 & 3.1658 & 1.7582 & 0.6345 & 3.1658 & 55.8628 & 0 & 55.8628 & P15 \\
\hline 1.7582 & 55.8628 & 1.7582 & 0.6345 & 55.8628 & 55.8628 & 0 & 55.8628 & P15 \\
\hline 1.7582 & 0.6345 & 1.7582 & 0.6345 & 0.6345 & 55.8628 & 3.1658 & 55.8628 & P15 \\
\hline 1.7582 & 3.1658 & 1.7582 & 0.6345 & 3.1658 & 55.8628 & 0 & 55.8628 & P15 \\
\hline 1.3383 & 3.4765 & 1.3383 & 1.3383 & 3.4765 & 51.3403 & 0 & 51.3403 & P16 \\
\hline 1.3383 & 3.4765 & 1.3383 & 1.3383 & 3.4765 & 51.3403 & 0 & 51.3403 & P16 \\
\hline 1.3383 & 1.3383 & 1.3383 & 1.3383 & 1.3383 & 51.3403 & 1.3383 & 51.3403 & P16 \\
\hline 1.3383 & 3.4765 & 1.3383 & 1.3383 & 3.4765 & 51.3403 & 0 & 51.3403 & P16 \\
\hline
\end{tabular}


features for both face and fingerprint for individuals (10-16).

\section{EXPERIMENT AND RESULTS}

Precise results of identification process generated by Dis-Eigen algorithm within the focal point of multirepresentation analysis for an individual's face-fingerprint. An enhancement level of an individual's face-fingerprint for Dis-Eigen feature-based fusion data utilization is proven in this work.

In this study, the proposed Dis-Eigen algorithm is Dis-Eigen feature-based fusion. Datasets with different numbers of both test and other train are used in this work, precisely two kinds of examples are established, The first adopt individual datasets with a split percentage of $60 \%$ as training and $40 \%$ for testing. The other has a split percentage of $80 \%$ as training and $20 \%$ as testing. The implementation of training is demonstrated using NaiveBayes, RandomForest, RandomForest, and J48

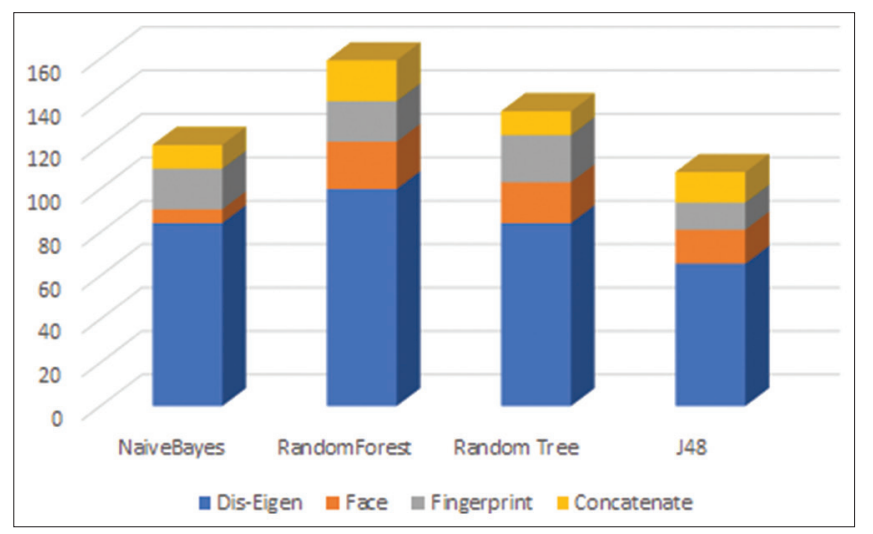

Fig. 4. Percentage of $60 \%$ training and $40 \%$ of testing.

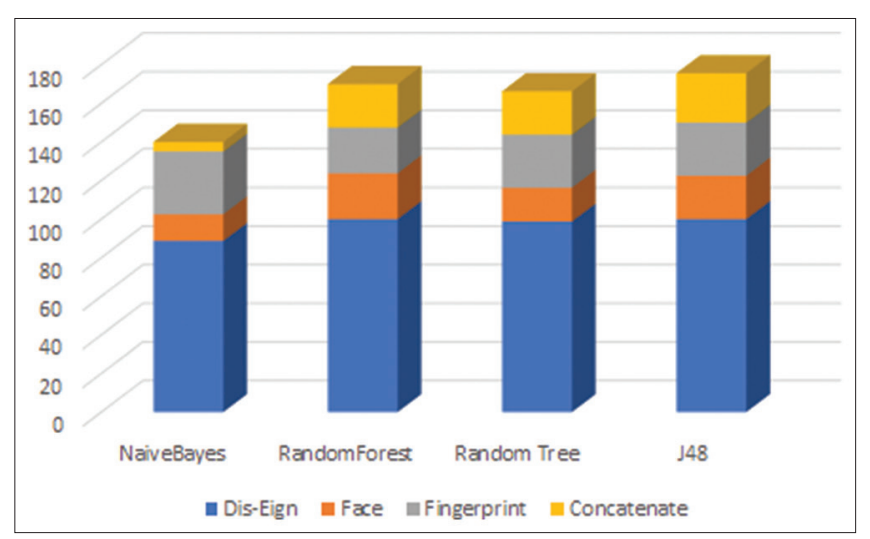

Fig. 5. All methods for eight (8) folds Cross. along with folds cross-validation of eight (8) and ten (10). In this experiment, the data sets comprise 160 data which are broken down into two categories with 20 individuals. As presented in Tables 4-7 along with Figs. 4 and 5.

Tables 4 and 5 show the performance of Dis-Eigen, face, fingerprint, and Concatenate features for the eight classifiers for the two experimental analysis setup. In average, the performance of Dis-Eigen feature for all classifiers has

\begin{tabular}{|c|c|c|c|c|}
\hline Methods & Naivebayes & $\begin{array}{c}\text { Random } \\
\text { forest }\end{array}$ & $\begin{array}{l}\text { Random } \\
\text { tree }\end{array}$ & J48 \\
\hline Dis-Eigen & 84.37 & 100 & 84.37 & 65.62 \\
\hline Face & 6.25 & 21.78 & 18.75 & 15.62 \\
\hline Fingerprint & 18.75 & 18.75 & 21.75 & 12.5 \\
\hline Concatenate & 10.93 & 18.75 & 10.93 & 14.06 \\
\hline
\end{tabular}

\begin{tabular}{|c|c|c|c|c|}
\hline Methods & NaiveBayes & RandomForest & $\begin{array}{l}\text { Random } \\
\text { Tree }\end{array}$ & J48 \\
\hline Dis-Eigen & 87.5 & 100 & 100 & 100 \\
\hline Face & 25 & 31.25 & 25 & 18.75 \\
\hline Fingerprint & 12.5 & 18.75 & 12.5 & 6.25 \\
\hline Concatenate & 15.62 & 18.75 & 12.5 & 15.62 \\
\hline
\end{tabular}

TABLE 6: Provide the accuracy for Dis-Eigen feature-based fusion with all Methods for eight (8) folds Cross-Validation

\begin{tabular}{lcccc}
\hline Methods & NaiveBayes & RandomForest & $\begin{array}{c}\text { Random } \\
\text { Tree }\end{array}$ & J48 \\
\hline Dis-Eigen & 88.75 & 100 & 98.75 & 100 \\
Face & 13.75 & 23.75 & 17.5 & 22.5 \\
Fingerprint & 32.5 & 23.75 & 27.5 & 27.5 \\
Concatenate & 5 & 22.5 & 22.5 & 25.62 \\
\hline
\end{tabular}

TABLE 7: Provide the accuracy for Dis-Eigen feature-based fusion with all Methods for ten (10) folds Cross-Validation

\begin{tabular}{lcccc}
\hline Methods & NaiveBayes & RandomForest & $\begin{array}{c}\text { Random } \\
\text { Tree }\end{array}$ & J48 \\
\hline Dis-Eigen & 91.25 & 100 & 100 & 98.75 \\
Face & 16.25 & 21.25 & 17.5 & 25 \\
Fingerprint & 33.75 & 25 & 22.5 & 28.75 \\
Concatenate & 4.37 & 25.62 & 20 & 20.62 \\
\hline
\end{tabular}


succeeded as the heights performance with $90.23 \%$ accuracy rate in average. This is followed by $20.3 \%$ for face rule, 14.64\% for Concatenate, and $15.21 \%$ for Fingerprint.

The Dis-Eigen algorithm has presented the best performance accuracy of $97.18 \%$ in average in Tables 6 and 7 for all classifiers with eight and ten fold cross validation environment setup. Though, six fusion and non-fusion algorithm have achieved quite a lower average performance of $19.68 \%$ for Face rule, 27.65\% for Fingerprint and 18.20\% for Concatenate. This is a very poor performance in comparison toward the performance of Dis-Eigen features. This has shown that Dis-Eigen algorithm significantly increased their classification performance.

As referred, Tables 4-7 and Figs. 4 and 5 presented the overall results of the various methods, it is a noticeable sign that the Dis-Eigen feature-based fusion has the optimum accuracy than face or fingerprint individually besides the Concatenate data. The preferable outcome achieves the applied improvement to the features which are individually represented through Dis-Eigen feature-based fusion.

\section{CONCLUSION}

The Dis-Eigen feature-based fusion algorithm has been proposed in this work as an attempt for multi-model biometric system improvement for individuality in facefingerprint identification.

The proposed algorithm converts the multi-representations of individual features into a uni-representation with the technique of the Dis-Eigen algorithm. Generalized features of an individual have been presented significantly. The new approach has been evaluated and beard comparison with the conventional one with regard to similarity measurement. According to this, an individuals' face- fingerprints were identified. Resultant scrutinization has been made. The outcome feature from the Dis-Eigen feature application is systematic and more informative as experimental resultants of the proposed approach show $93.70 \%$ identification rate with feature-level fusion multi-biometric individual identification. Furthermore, a particular improvement in system performance accuracy is achieved.

\section{REFERENCES}

[1] M. Al-Ani and K. Al-Baset. "Efficient Watermarking based an Robust Biometric Features." IRACST-Engineering Science and Technology: An International Journal, vol. 3, pp. 529-534, 2013.
[2] M. S. Al-Ani and M. A. Rajab. "Biometrics hand geometry using discrete cosine transform (DCT)." Science and Technology, vol. 3, no. 4, pp. 112-117, 2013.

[3] M. Al-Ani and S. Nejrs. "Efficient biometric iris recognition based on iris localization approach." UHD Journal of Science and Technology, vol. 3, pp. 24-32, 2020.

[4] Z. A. Kakarash, D. F. Abd, M. Al-Ani, G. A. Omar and K. Mohammed. "Biometric Iris Recognition Approach Based on Filtering Techniques." 2019.

[5] M. S. Al-Ani, T. N. Muhamad, H. A. Muhamad and A. A. Nuri. "Effective Fingerprint Recognition Approach Based on Double Fingerprint Thumb." In: 2017 International Conference on Current Research in Computer Science and Information Technology (ICCIT), 2017.

[6] M. ShabanAl-Ani and W. M. Al-Aloosi. "Biometrics fingerprint recognition using discrete cosine transform (DCT)." International Journal of Computer Applicationsvol, 69, no. 6, pp. 44-48, 2013.

[7] O. H. A. Al-Ani. "Human identification based on thinning minutiae of fingerprint." Journal of Theoretical and Applied Information Technology, vol. 96, no. 17, pp. 5918-5929, 2018.

[8] M. S. Al-Ani. "A novel thinning algorithm for fingerprint recognition." International Journal of Engineering Sciences, vol. 2, no. 2, pp. 4348, 2013.

[9] R. S. Prasad, M. S. Al-Ani and S. M. Nejres. "An efficient approach for fingerprint recognition." International Journal of Engineering Innovation and Research, vol. 4, no. 2, pp. 307-313, 2015.

[10] B. O. Mohammed and S. M. Shamsuddin. "Feature Level Fusion for Multi-Biometric with Identical Twins." In: 2018 International Conference on Smart Computing and Electronic Enterprise (ICSCEE), 2018.

[11] B. O. Mohammed. "Fusion method with mean-discrete algorithm in feature level for identical twins identification." UHD Journal of Science and Technology, vol. 4, no. 2, pp. 141-150, 2020.

[12] B. O. Mohammed and Z. M. Hashim. "Individuality representation using multimodal biometrics with aspect unieted moment invariant for identical twins." Journal of Theoretical and Applied Information Technology, vol. 98, no. 12, pp. 2148-2157, 2020.

[13] B. O. Mohammed. "Mean-discrete algorithm for individuality representation." Journal of Al-Qadisiyah for Computer Science and Mathematics, vol. 13, no. 1, pp. 1-10, 2021.

[14] Q. K. Al-Shayea, M. S. Al-Ani and M. S. A. Teamah. "The effect of image compression on face recognition algorithms." International Journal of Computer and Network Security, vol. 2, no. 8, pp. 56-60, 2010.

[15] M. S. Al Ani. "Face Recognition Approach Based on WaveletCurvelet Technique." Signal Image Process, vol. 3, no. 2, pp. 2131, 2012.

[16] R. S. Prasad, M. S. Al-Ani and S. M. Nejres. "An efficient approach for human face recognition." International Journal of Advanced Research in Computer Science and Software Engineering, vol. 5, no. 9, pp. 133-136, 2015.

[17] M. A. A. Qeethara Al-Shayea. "Biometric face recognition based on enhanced histogram approach." International Journal of Communication Networks and Information Security, vol. 10, no. 1, pp. 148-154, 2018.

[18] O. H. Ahmed, J. Lu, Q. Xu and M. S. Al-Ani. "Face recognition based rank reduction SVD approach." The ISC International Journal of Information Security, vol. 11, no. 3, pp. 39-50, 2019.

[19] R. S. Prasad, M. S. Al-Ani and S. M. Nejres. "Human identification 
via face recognition: Comparative study." IOSR Journal of Computer Engineering, vol. 19, no. 3, pp. 17-22, 2017.

[20] M. S. Al Ani and A. S. Al Waisy. "Multi-view face detection based on kernel principal component analysis and kernel support vector techniques." International Journal on Soft Computing, vol. 2, no. 2, pp. 1-13, 2011.

[21] S. M. Nejrs and M. S. Al-Ani. "Face image classification based on feature extraction." Solid State Technology, vol. 63, no. 6, pp. 13515-13526, 2020.

[22] M. S. Al-Ani. "Happiness measurement through classroom based on face tracking." UHD Journal of Science and Technology, vol. 3, no. 1, pp. 9-18, 2019.

[23] Sriram J and Jacob J. "Smart EVM based on face and fingerprint recognition." IJRASET, vol. 8, no. 8, pp. 1606-1610, 2020.

[24] M. Szymkowski and K. Saeed. "A Multimodal Face and Fingerprint Recognition Biometrics System." In: Computer Information Systems and Industrial Management. Springer International Publishing, Cham, pp. 131-140, 2017.

[25] D. C. K. Divyakant and T. Meva. "Performance Measurement of
Face and Fingerprint Recognition System." In: RK University First International Conference On Research and Entrepreneurship, 2016.

[26] M. Abd Al Rahim, W. El-Shafai, E. S. M. El-Rabaie, O. Zahran and F. E. Abd El-Samie. "Comb filter approach for cancelable face and fingerprints recognition." Menoufia Journal of Electronic Engineering Research, vol. 28, no. 1, pp. 89-94, 2019.

[27] G. S. G. Anjaneyulu and V. Jalaja. "Novel Authentication Process of the Smart Cards Using Face and Fingerprint Recognition." In: Advances in Automation, Signal Processing, Instrumentation, and Control. Springer, Singapore, pp. 2547-2556, 2021.

[28] B. O. Mohammed and S. M. Shamsuddin. "Twins multimodal biometric identification system with aspect united moment invariant." Journal of Theoretical and Applied Information Technology, vol. 95, no. 4, pp. 788-803, 2017.

[29] B. O. Mohammed and S. M. Shamsuddin. "A multimodal biometric system using global features for identical twins identification." Journal of Computational Science, vol. 14, no. 1, pp. 92-107, 2018. 